\title{
El uso de biofertilizantes en el cultivo de frijol (phaseolus vulgaris 1.)
}

The use of biofertilizers in bee cultivation (phaseolus vulgaris 1.)

\begin{abstract}
Alvarado Gómez Luis Carlos ${ }^{1}$, Eduardo Manuel Graillet Juárez ${ }^{1}$, Marina Martínez Martínez ${ }^{1}$, José Antonio Fernández Figueroa ${ }^{1}$ y Ronnie de Jesús Arieta Román ${ }^{1}$
\end{abstract}

${ }^{1}$ Facultad de Ingeniería en Sistemas de Producción Agropecuaria. Universidad Veracruzana.

${ }^{凶}$ Autor para correspondencia: luiscarlos510@hotmail.com

Recibido: $15 / 07 / 2017$

Aceptado: 10/12/2017

\section{RESUMEN}

El frijol es un cultivo estratégico para México, representa una importante fuente de ocupación e ingreso en la economía campesina y en la dieta, representa la única y principal fuente de proteínas, para amplias sectores de la población, además es un alimento con importantes propiedades nutracéuticas. En el sur de Veracruz, se obtienen rendimientos promedio de 700 kilogramos por hectárea, esta baja productividad obedece principalmente al uso de materiales criollos regionales de limitada capacidad productiva y susceptible a enfermedades y un manejo inadecuado del cultivo, por lo que el objetivo de este estudio fue determinar el potencia de rendimiento de cuatro genotipos de frijol (Phaseolus vulgaris L.), su respuesta a la fertilización mineral y al uso de biofertilizantes, en el municipio de Catemaco, Veracruz. Los rendimientos de grano $\mathrm{ha}^{-1}$ indican que no hubo diferencia entre genotipos, con rendimientos promedio de casi $2 \mathrm{t} \mathrm{ha}^{-1}$, casi el triple de los promedios de la región. El uso de la fórmula 40-40-40, casi duplica el rendimiento del testigo absoluto, el uso de biofertilizantes también incrementó el rendimiento y la interacción de primer orden entre genotipos y fertilización mineral, resulto significativa estadísticamente.

Palabras clave: Frijol, Biofertilizantes, Veracruz, Genotipo.

\begin{abstract}
ABSTRAC
The bean is a strategic crop for Mexico, it represents an important source of occupation and income in the peasant economy and in the diet, it represents the only and main source of proteins for large sectors of the population, it is also a food with important nutraceutical properties . In the south of Veracruz, average yields of 700 kilograms per hectare are obtained, this low productivity is mainly due to the use of regional criollo materials of limited productive capacity and susceptible to diseases and inadequate crop management, so the objective of this study was to determine the yield potency of four genotypes of bean (Phaseolus vulgaris L.), its response to mineral fertilization and the use of biofertilizers, in the municipality of Catemaco, Veracruz. Grain yields ha-1 indicate that there was no difference between genotypes, with average yields of almost $2 \mathrm{t}$ ha-1, almost triple the averages of the region. The use of the formula 40-40-40, almost doubles the yield of the absolute control, the use of biofertilizers also increased
\end{abstract}


the yield and the first-order interaction between genotypes and mineral fertilization, was statistically significant.

Keywords: Bean, Biofertilizers, Veracruz, Genotype.

\section{INTRODUCCIÓN}

El frijol (Phaseolus vulgaris L.) es la especie más importante para el consumo humano, entre las leguminosas de grano alimenticias. Por su alto contenido de proteínas, minerales esenciales, fibra y vitaminas del complejo B, tiene gran importancia en la dieta de los mexicanos, desde el punto de vista nutricional, siendo un producto clave en la seguridad alimentaria (Voysest, 2000). En la zona sur de Veracruz, se obtienen rendimientos promedio de 700 kilogramos por hectárea, esta baja productividad obedece principalmente al uso de materiales criollos regionales de limitada capacidad productiva y susceptible a enfermedades y un manejo inadecuado del cultivo, por lo que el objetivo de este estudio fue determinar el potencia de rendimiento de cuatro genotipos de frijol (Phaseolus vulgaris L.), su respuesta a la fertilización mineral y al uso de biofertilizantes, en el municipio de Catemaco, Veracruz.

\section{MATERIALES Y MÉTODOS}

El experimento se estableció en terrenos de un agricultor cooperante en la comunidad Cerro del Nopo, Ejido Catemaco, municipio de Catemaco, Veracruz. Ubicada en las coordenadas geográficas $18^{\circ} 24^{\prime} \mathrm{LN}$ y los $95^{\circ} 6^{\prime} \mathrm{LO}$ y a los $902 \mathrm{msnm}$. El clima de la región de acuerdo a la clasificación de Köppen, modificado por Enriqueta García, (1987) es: (Am) Monzónico, con una temperatura promedio $26{ }^{\circ} \mathrm{C}$ y precipitación pluvial media anual de 2,125 mm y Suelos Andosoles. Se utilizó un arreglo factorial de parcelas subdivididas, bajo un diseño de bloques completos al azar con cuatro repeticiones. Los factores y niveles estudiados fueron, los genotipos de frijol negro Comapa, negro Inifap, negro Tropical y negro Veracruz, las fórmulas de fertilización 00-00-00 y 40-4040, kilogramos de nitrógeno, fosforo y potasio, respectivamente, sin y con biofertilizantes. El Biofertilizante utilizado, fue a base de micorrizas Glomus intradices y Azospirilum brasilenses. El experimento se inició con el trazo de la parcela experimental, sobre el terreno, delimitando las parcelas y calles con rafia convencional. Posteriormente se hizo la selección y asignación de los tratamientos, en las parcelas experimentales. La preparación del suelo consistió en un chapeo y la aplicación de Glifosato ${ }^{\circledR}$ a dosis de $2 \mathrm{~L} \mathrm{ha}^{-1}$. La siembra se realizó el 15 de febrero del 2015, a una distancia entre surcos de $40 \mathrm{~cm}$ y entre plantas de $20 \mathrm{~cm}$ y con tres plantas por mata obteniendo una densidad de siembra de 375,000 plantas por hectárea. Las unidades experimentales fueron seis surcos de 10 metros de largo con una separación entre bloques de un metro. La parcela útil estuvo constituida por los cuatro surcos centrales, eliminando un metro de cabecera. El experimento se cosechó el 24 de mayo del 2016. Las variables evaluadas fueron, número de vainas por planta, número de granos por vaina, peso de grano por planta y rendimiento de grano por hectárea, ajustado al 14\% de humedad. Para el análisis estadístico, se utilizó la técnica del Análisis de Varianza, y la comparación de medias por el método de Tukey 0.05 con el paquete Statistics.

\section{RESULTADOS Y DISCUSIÓN}

Número de vainas por planta. Al realizar el Análisis de Varianza y la comparación de medias por el método de Tukey, para la variable número de vainas por planta, el genotipo negro Veracruz, superó a las demás genotipos, con diferencia significativa. En relación a la fertilización mineral, se obtuvo una diferencia 
altamente significativa, al utilizar la fórmula 4040-40 casi se duplicó el número de vainas en relación a la no fertilización. Con el uso de biofertilizantes se observó una diferencia significativa, para esta variable.

Número de granos por vaina. Para la variable número de granos por vaina, los resultados no mostraron diferencias estadísticas, para ninguno de los factores evaluados, estos resultados coincide con Checa-Coral et al. (2011), quienes en estudios realizados en nueve líneas homocigóticas de frijol y cuatro localidades del departamento de Nariño en Colombia, determinaron que el número de granos por vaina, es una característica de alta heredabilidad (78.2 $\%)$, por tanto depende principalmente de factores genéticos.

Peso de grano por planta. Para la variable peso de grano por planta, no se observaron diferencias significativas entre genotipos de frijol. En relación a la fertilización mineral, se obtuvo una diferencia altamente significativa, al utilizar la fórmula 40-40-40 casi se duplicó el peso de grano por planta, en relación al testigo absoluto. La aplicación de biofertilizantes mostró un aumento y diferencias significativas, para esta variable.

Rendimiento de grano por ha. El rendimiento del frijol (Phaseolus vulgaris L.) por hectárea, es un carácter que depende de factores genéticos, edafoclimáticos y de manejo y es considerado un carácter de baja heredabilidad (36\%) (ChecaCoral et al., 2011). Esto significa que depende más de factores de clima, suelo y manejo que del componente genético. Por tanto mientras más nos acerquemos al manejo adecuado, será menos probable que encontremos diferencias entre variedades mejoradas. Esto ocurrió en este estudio. No se observaron diferencias significativas entre las variedades evaluadas. Al utilizar la fórmula de fertilización 40-40-40 casi se duplicó el rendimiento por hectárea, en relación al testigo absoluto y también se encontró diferencia significativa para el uso de biofertilizantes, (Cuadro 1).

Cuadro 1. Comparación de medias para las variables, número de vainas por planta, número de granos por vaina, peso de grano por planta y rendimiento en kilogramos por hectárea del experimento "Genotipos de frijol (Phaseolus vulgaris L.), fertilización mineral y uso de biofertilizantes en Catemaco, Veracruz".

\begin{tabular}{|c|c|c|c|c|}
\hline Factor & Vainas pl ${ }^{-1}$ & Granos vaina $^{-1}$ & $\begin{array}{c}\text { Peso de grano } \\
\mathrm{pl}^{-1}(\mathrm{~g})\end{array}$ & $\begin{array}{c}\text { Rendimiento } \\
\left(\mathrm{kg} \mathrm{ha}^{-1}\right)\end{array}$ \\
\hline Negro Comapa & $6.9 \mathrm{~b}$ & $4.6 \mathrm{a}$ & $8.3 \mathrm{a}$ & $1,867 \mathrm{a}$ \\
\hline Negro Inifap & $5.7 b$ & $4.6 \mathrm{a}$ & $7.4 \mathrm{a}$ & $1,665 \mathrm{a}$ \\
\hline Negro Tropical & $6.0 \mathrm{~b}$ & $4.7 \mathrm{a}$ & $6.4 \mathrm{a}$ & $1,440 \mathrm{a}$ \\
\hline Negro Veracruz & $8.0 a$ & $4.6 \mathrm{a}$ & $8.6 \mathrm{a}$ & $1,935 \mathrm{a}$ \\
\hline 00-00-00 & $5.7 b$ & $4.3 \mathrm{a}$ & $5.6 \mathrm{~b}$ & $1,260 \mathrm{~b}$ \\
\hline $40-40-40$ & $9.4 a$ & $4.8 \mathrm{a}$ & $9.8 \mathrm{a}$ & $2,205 \mathrm{a}$ \\
\hline Sin biofertilizante & $7.0 \mathrm{~b}$ & $4.6 \mathrm{a}$ & $7.2 \mathrm{~b}$ & $1,620 \mathrm{~b}$ \\
\hline Con biofertilizante & $8.1 a$ & $4.8 \mathrm{a}$ & $8.2 \mathrm{a}$ & $1,845 \mathrm{a}$ \\
\hline $\mathrm{CV}$ & $22.5 \%$ & $18.2 \%$ & $23.5 \%$ & $25.2 \%$ \\
\hline
\end{tabular}

\section{Interacción entre genotipos y fertilización mineral}

La interacción entre factores es un comportamiento diferencial, de un factor, dentro de los niveles del otro, en las gráficas se identifica al tener líneas que no son paralelas entre ellas y solo se puede identificar en los experimentos factoriales. En fenómenos biológicos se ha identificado que muchos factores interactúan entre sí. En Genética se 
utiliza la interacción genotipo x ambiente, que es parte del modelo aditivo y multiplicativo, para respuestas fenotípicas. En este experimento se observó una interacción altamente significativa entre genotipos y fertilización mineral. Cuando existe integración significativa, se dice que no hay un mejor genotipo, sino que hay un mejor genotipo para ciertas condiciones específicas, en este caso sin fertilización mineral, el genotipo negro Veracruz superó a los demás y con la fórmula 40-40-40 negro Veracruz y negro Comapa, tuvieron los mejores rendimientos, como se observa en la Figura 1.

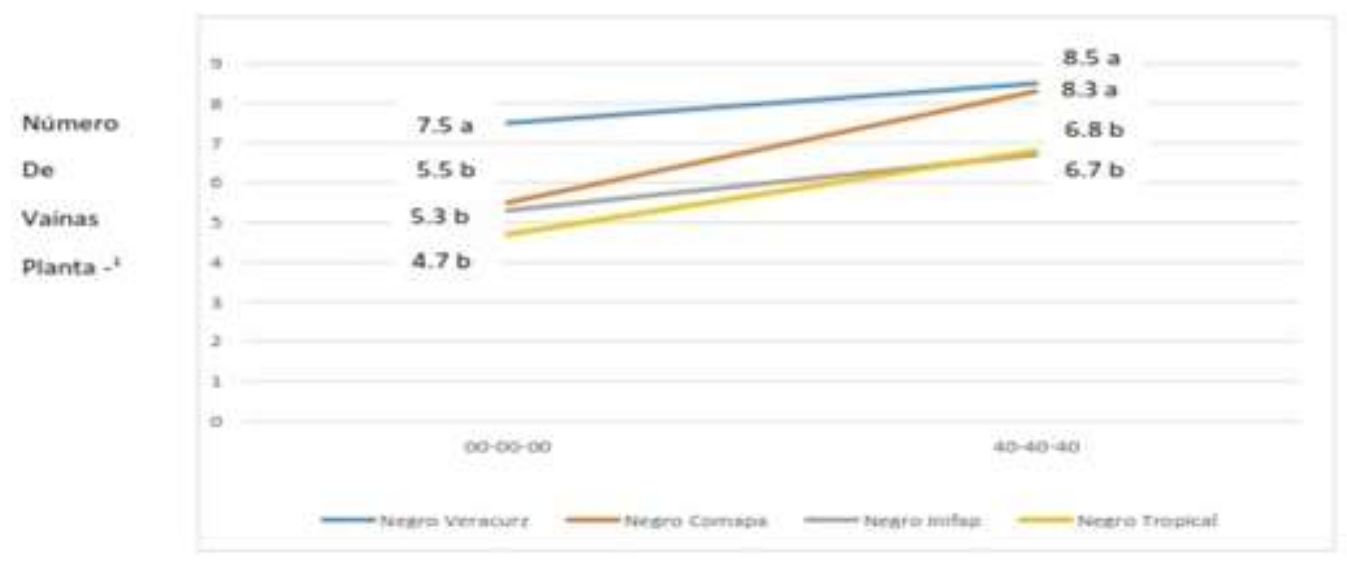

Figura 1. Interacelón entre genotipos y fertillzación mineral, para la variable nùmero de valnas por planta, en el experimento "Genotipos de frijol (Phaseolus vulgaris L.). fertilizacion mineral y uso de blofertiltzantes en Catemaco, Veracruz".

\section{CONCLUSIONES}

1. Para genotipos solo se observó diferencia significativa para la variable vainas por planta.

2. La fertilización con la fórmula 40-40-40, casi duplicó el número de vainas por planta, el peso de grano por planta y el rendimiento por ha.

3. El uso del biofertilizante, logró incrementos del $15 \%$, con diferencias significativas, para el número de vainas por planta, el peso de grano por planta y el rendimiento por ha.

4. La interacción de primer orden entre genotipos y fertilización mineral, resulto significativa estadísticamente.

\section{LITERATURA CITADA}

Checo-Coral, O. E., V. M. Yama-Escobar y S. M. Fuel-Tovar. 2011. Evaluación por componentes de rendimiento de nueve genotipos y un testigo de frijol arbustivo Phaseolus vulgaris L. Revista de Ciencias Agrícolas. 28 (1): 73-90.

García, E. 1987. Modificaciones al Sistema de Clasificación Climática de Köppen (para adaptarlo a las condiciones de la República Mexicana). 4a ed. Universidad Nacional Autónoma de México. México, D. F. 130 p.

Voysest, O. 2000. Mejoramiento Genético del fríjol (Phaseolus vulgaris L.) legado de variedades de América Latina 1930-1999. Cali, Colombia. Centro Internacional de Agricultura tropical CIAT. 
Copyright (c) 2017 Luis Carlos Alvarado Gómez, Eduardo Manuel G raillet Juárez, Marina Martinez Martinez, José Antonio

Fernández Figueroa y Ronnie de Jesús Arieta Rom án

(c) (9)

Este tex to está protegido por una licencia licencia Creative Commons 4.0 .

\begin{abstract}
Usted es libre para Compartir —copiar y redistribuir el material en cualquier medio o form ato- y Adaptar el documento -remezdar, transformar y crear a partir del material- para cualquier propósito, incluso para fines comerciales, siempre que cumpla la condición de:

Atribución: Usted debe dar crédito a la obra original de manera adecuada, proporcionar un enlace a la licencia, e indicar si se han realizado cam bios. Puede hacerlo en cualquier form a razonable, pero no de form a tal que sugiera que tiene el apoyo del licenciante o 10 recibe por el uso que hace de la obra.

Resumenclicicencia - Textocompletodelalicencia
\end{abstract}

